\title{
El patrimonio ferroviario, un vehículo para la valoración del territorio. Rehabilitación y re-funcionalización de talleres en el Noroeste de la Provincia de Buenos Aires
}

\author{
Melina YULN* \\ Universidad Nacional del Noroeste de la Provincia de Buenos Aires, Argentina \\ Federico Montecelli*** \\ Universidad Nacional del Noroeste de la Provincia de Buenos Aires, Argentina \\ Silvina Cecilia Carrizo**** \\ Universidad Nacional del Noroeste de la Provincia de Buenos Aires, Argentina
}

\begin{abstract}
Resumen: El ferrocarril resultó determinante para el crecimiento y desarrollo de los pueblos bonaerenses, en el entresiglos XIX-XX. La instalación de talleres ferroviarios fortaleció la actividad económica de las localidades involucradas y la identidad local quedó íntimamente ligada a la actividad ferroviaria. Hacia finales de 1970 la política nacional argentina privilegió el desarrollo vial y el ferrocarril entró en una etapa de abandono y desmantelamiento. La actividad decayó y se iniciaron procesos de des-territorialización que se aceleraron durante 1990, con la privatización de los ferrocarriles. Las comunidades afectadas buscaron alternativas para re-dinamizar la vida local, mediante la re-funcionalización y puesta en valor del patrimonio ferroviario. Los complejos de talleres de Junín y Mechita, en el noroeste de la provincia de Buenos Aires, fueron rehabilitados con proyectos impulsados por municipios, instituciones y organizaciones no gubernamentales. La hipótesis de este trabajo es que el patrimonio ferroviario puede ser vehículo de re-territorialización en localidades argentinas.
\end{abstract}

Palabras Clave: patrimonio- ferrocarril — re-funcionalización — re-territorialización — desarrollo local

Railway heritage: a vehicle to value the territory. Rehabilitation and re-functionalization of railway workshops in the Northwest of Buenos Aires Province.

Abstract: The railroad was decisive for the growth and development of the Buenos Aires towns, in between siècle's XIX-XX. Local economic activity was strengthened in towns where railway workshops were installed, and the local identity was closely linked to the railway activity. Towards the end of 1970 Argentine national policy favored road development and the railroad entered a stage of abandonment and dismantling. The activity declined and began processes of de-territorialization that accelerated during 1990, with the privatization of the railways. The affected communities sought alternatives to revitalize local life, through the redefinition and enhancement of the railway heritage. In the northwest of Buenos Aires province, Junín and Mechita railway workshops were rehabilitated with projects promoted by municipalities, institutions and non-governmental organizations. The hypothesis of this article is that railway heritage may be a vehicle for reterritorialization in Argentinean towns. Keywords: heritage - railroad - refunctionalization - reterritorialization - local development

\footnotetext{
* Docente Investigadora, TEAM-UNNOBA; E-mail: melinayuln@yahoo.com.ar

** Fedem_v18@hotmail.com

****silcarrizo@conicet.gov.a
} 


\section{Introducción}

La rehabilitación y re-funcionalización de edificios y complejos edilicios abandonados por procesos de desindustrialización o por cambios de su función original es una práctica instalada en Europa y EE.UU., que posteriormente se ha trasladado a Latinoamérica (Capel, 1996; del Pozo, 2002). En las discusiones ligadas al proceso de reutilización de construcciones se destacan dos funciones complementarias: por un lado, el rescate del patrimonio histórico y por otro, la promoción del desarrollo local (Prats, 2003; Feria Toribio, 2010). Para éste la actividad turística aparece como opción relevante (Troncoso y Almirón 2005; Almirón et al, 2006) a la vez que pone en relieve la valoración del territorio como patrimonio (Di Méo, 2007; Sabaté, 2014).

En este contexto entran en juego la valorización de la historia y la identidad de las comunidades, de manera que se incluye no sólo al patrimonio edilicio, sino también al patrimonio productivo rural, minero o de infraestructura (Sabaté y del Pozo, 2010). La arquitectura y la infraestructura ferroviaria representan un legado patrimonial histórico y cultural, cuya posibilidad de explotación abre nuevas perspectivas para las comunidades que deciden re-significar su antiguo uso (Dias y Galvao, 2010).

En Argentina el ferrocarril se extendió durante el entresiglos XIX-XX, llevando consigo la infraestructura y la arquitectura que caracterizarían a esta red de escala nacional y alto impacto territorial. Hacia finales de la década de 1970, las políticas nacionales privilegiaron el transporte vial y esto contribuyó al abandono y desmantelamiento del ferrocarril. La actividad ferroviaria decayó y muchas personas debieron cambiar de trabajo y /o emigrar. Durante la década de 1990, con la privatización de los ferrocarriles, un proceso de des-territorialización tomó celeridad.

Por des-territorialización se entiende a una fase de debilitamiento de la relación individual o colectiva con el espacio considerado como apropiado (Brunet, Thery, 1998). Esa pérdida de territorialidad puede conducir a su reinvención -por la aparición de otra forma de relacionamiento al lugar- y un proceso de re-territorialización. Un evento político como la reforma del Estado argentino en los años 1990, desencadenó profundas transformaciones socioeconómicas. En los territorios especializados -como los ferroviarios pero también en otros como los petroleros- esto puede significar pérdida de identidad. Las actividades que suplanten a aquella desaparecida, irán modelando una nueva identidad (Figura 1).

Figura 1. Ferrocarriles y (des) territorialización.

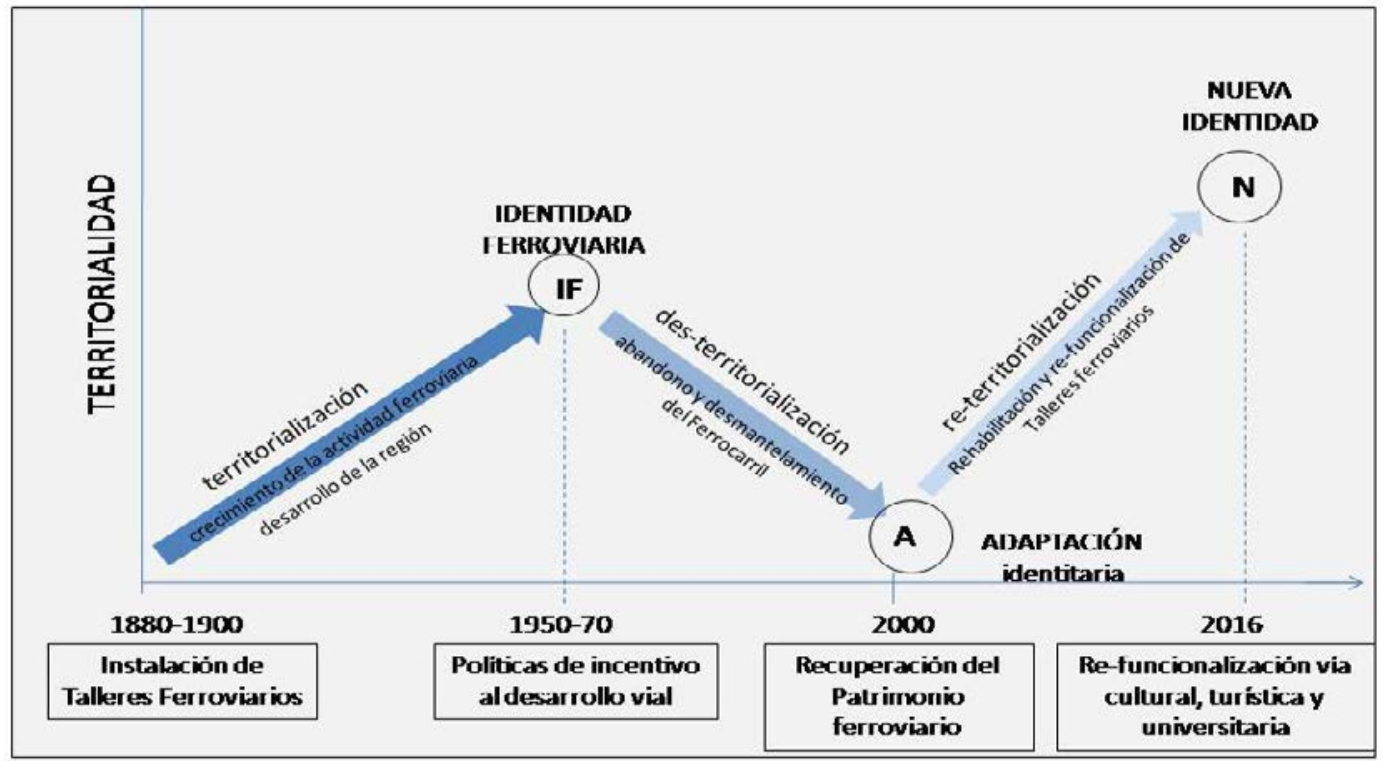

Fuente: Autores 
Diversos complejos edilicios de origen ferroviario, que perdieron su función original, han sido recuperados. Las rehabilitaciones -impulsadas por municipios, instituciones o grupos comunitariosabrieron un espectro amplio de posibilidades, donde las re-funcionalizaciones se traducen en un extenso abanico de usos: proyectos museísticos (Administración de Infraestructuras Ferroviarias del Estado); propuestas de infraestructura sostenible (Ferrari, 2013; Franco y Dillon, 1998); turismo cultural; sedes educativo-académicas; cooperativismo (Pompei, 2001).

El propósito de este artículo es explorar las posibilidades que ofrece la valorización del patrimonio como vehículo para el desarrollo territorial. Este trabajo se enmarca en un proyecto de investigación con sede en la Universidad Nacional del Noroeste de la provincia de Buenos Aires (UNNOBA). La investigación se origina a partir del estudio del patrimonio arquitectónico del Noroeste de Buenos Aires (NOBA). Para ello se recopilaron datos documentales, históricos, arquitectónicos y fotográficos y se realizaron entrevistas con actores locales. Además se contó con el aporte del proyecto cultural "Catálogo de Bienes Patrimoniales del partido de Bragado" que recopila información local y promueve la puesta en valor del patrimonio. El trabajo de campo permitió profundizar el estudio de las actividades productivas y culturales realizadas, con el fin de conocer no sólo espacios físicos pertenecientes al ferrocarril, sino también la historia e identidad del lugar. En este artículo se analiza el patrimonio ferroviario y su nueva puesta en valor en Junín, cabecera del partido homónimo, y en Mechita, localidad del partido de Bragado (Figura 2).

El texto se estructura en tres partes: la primera aborda los vínculos históricos de las localidades de Junín y Mechita con la actividad ferroviaria; la segunda parte trata sobre los sitios que componen los complejos ferroviarios rehabilitados y la tercera parte presenta las diferentes alternativas para su re-funcionalización.

Figura 2. Mapa de localización del área de estudio
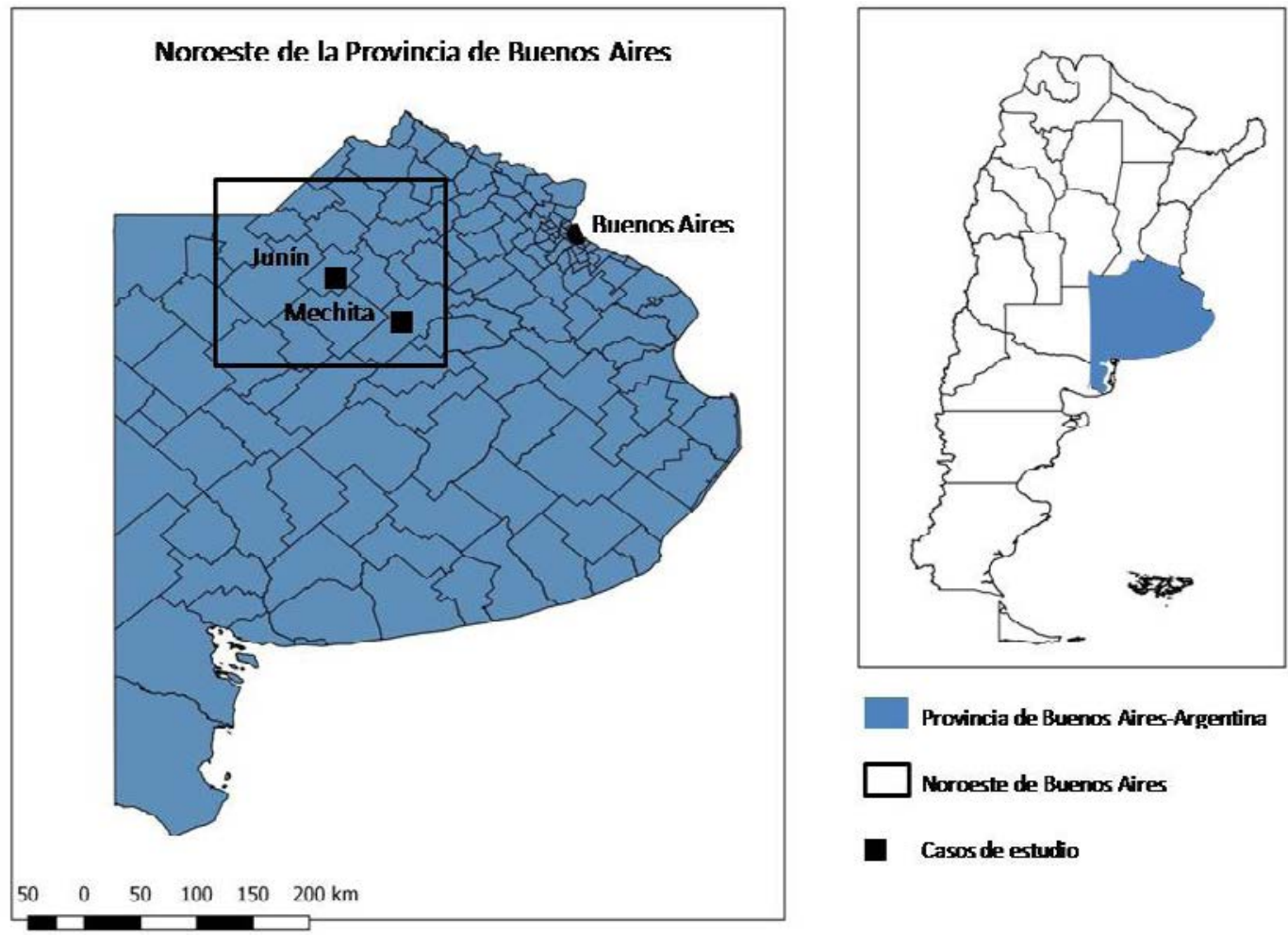

Provincia de Buenos Aires-Argentina

Fuente: Autores 


\section{El ferrocarril en el NOBA}

El tren tuvo un rol clave para la sociedad y la economía argentina entre finales del siglo XIX y principios del XX, no solo para el transporte de pasajeros y carga sino en el poblamiento y dinamización productiva del vasto territorio nacional. Su llegada a distintos puntos del país produjo cambios en la vida y en las perspectivas de las comunidades vinculadas. Las industrias pudieron ampliar sus mercados en sitios más lejanos; la explotación y provisión de materias primas se vio facilitada y bajaron los costos. Se aceleró la dinámica productiva y comercial; y se incrementaron las construcciones edilicias e infraestructura.

A partir de 1880 se dio la mayor expansión ferroviaria. La llegada a centros agrícolas significó un cambio económico considerable para el transporte de carga, particularmente para los molinos, cuya producción podía llegar rápidamente a Buenos Aires. Evitaba viajes en carretas de más de una semana de duración (Gómez y Schvarzer, 2007). Favoreció el crecimiento de la población en los pueblos, en gran parte por la llegada masiva de inmigrantes al país entre 1880 y 1910.

En la Provincia de Buenos Aires en 1870 existían cuatro líneas ferroviarias: hacia el norte, hasta Tigre; hacia el oeste, hasta Chivilcoy; hacia el sur, hasta Chascomús; y hacia Ensenada, hasta el puerto. La línea del oeste, que alcanzó la región NOBA, fue el primer servicio ferroviario en traspasar el área metropolitana. Hasta entonces el ferrocarril llegaba a Moreno -en las afueras de la Capital Federal- y funcionaba como un tren suburbano. El Ferrocarril del Oeste (FCO) fue la primera línea ferroviaria de escala nacional. Perteneció a la Provincia de Buenos Aires desde su apertura -en 1854- hasta 1889, cuando pasó a manos de una compañía extranjera, la Buenos Aires Western Railway Limited, de capitales ingleses. Esta línea ferroviaria llegó hasta Chivilcoy en 1866, y ese ramal se extendería hasta Alberti y Bragado, en 1877.

En 1884 el Ferrocarril Buenos Aires al Pacífico (BAP) llegó a Junín y en 1885, lo hizo el Ferrocarril Central Argentino (FCA), desde Pergamino (Scalabrini Ortiz, 1995). En 1886, se instaló un pequeño taller del BAP que creció rápidamente. Su función era mantener y reparar los trenes que, en gran medida, transportaban materias primas. Junín era entonces un pueblo que contaba con unos 1500 habitantes y cuya población rural alcanzaba los 3000 habitantes ${ }^{1}$. El proceso de instalación y crecimiento ferroviario en Junín coincide con el período "británico" de la explotación (1884-1947) y con el apogeo del modelo agroexportador. El vínculo entre el ferrocarril y la exportación agrícola de este período produjo un crecimiento exponencial de la población en los años subsiguientes, así como la definición de su configuración urbana a partir de las vías que atravesaban la trama de la ciudad. Entre 1890 y 1910 se construyeron edificios de envergadura, ligados a los talleres del ferrocarril BAP: en 1890 se construyeron los edificios de jefatura, talleres y construcciones utilitarias; en 1900 se construyó la estación y una vivienda y se estima que en 1910, se construyó la vivienda del Ingeniero Jefe Mecánico -el chalet York- junto con la oficina del departamento de mecánica y un importante número de edificios subsidiarios (Franco y Dillon, 1998). Con estas obras y actividades, hacia principios de 1900, 40\% de la población de Junín dependía del trabajo ferroviario, que constituyó la principal fuente de empleo y de recursos económicos de la ciudad y de su zona de influencia. El número de empleados superaba los 1500 y la población de la ciudad había alcanzado los 23.000 habitantes, debido en gran parte al arribo de familias de los nuevos trabajadores de los talleres. Los mismos se fueron radicando en distintos barrios que se conformaron en torno a las vías del ferrocarril. Los diferentes ramales originaron una serie de límites físicos en torno a los que se definieron núcleos habitacionales con características identitarias y socioeconómicas distintas (Montecelli et al, 2015).

En 1908, en el partido de Bragado, se terminó la construcción de talleres ferroviarios ${ }^{2}$ y de un depósito de locomotoras, sobre la línea del Ferrocarril Oeste a $10 \mathrm{~km}$ de la ciudad Bragado y a $17 \mathrm{~km}$ de Alberti. También se construyó una playa de maniobras y una colonia de viviendas para personal ferroviario. En 1910, se inauguró la "Estación Mechita"3. La localidad de Mechita como otras del interior, surgió por la instalación de la estación intermedia- para proveer agua alas calderas de las locomotoras a vapor y brindar servicios mecánicos-y creció a raíz del flujo de bienes y personas generado por la propia estación. La economía del pueblo se concentró casi exclusivamente en los talleres ferroviarios. El pueblo creció con la inmigración y en los primeros años del siglo XX 
surgió una intensa vida social, acompañada de la apertura de clubes e instituciones. Durante la década de 1950 la población llegó a unos cinco mil habitantes y las 118 viviendas de estilo inglés, construidas inicialmente, fueron rebasadas ampliamente. Circulaban entre seis y siete trenes diarios de pasajeros hacia y desde la Capital Federal y varios trenes diarios a la ciudad de Bragado para transportar operarios (Morello y Aguilar, 2006).

\section{Sitios patrimoniales rehabilitados}

A principios de la década de 1990, los ferrocarriles estatales fueron alcanzados por el proceso nacional de privatizaciones y reestructuraciones de grandes empresas de servicios. Tanto Junín como Mechita vieron reducida su actividad ferroviaria ${ }^{4}$. Los edificios del ferrocarril, que ocupan sitios significativos en ambas localidades, fueron rehabilitados posteriormente para desempeñar una nueva función. A continuación se detallan los sitios que componen los complejos ferroviarios que han sido re-habilitados, refuncionalizados o han recibido protección patrimonial.

a) En Junín:

\section{- Talleres:}

El tendido de las vías y sus construcciones subsidiarias generó cambios en la estructura urbana de Junín, propiciando el crecimiento de nuevos sectores y barrios. La ubicación de los ramales ferroviarios constituyeron una suerte de límite urbano que generó tres núcleos habitacionales independientes, con características identitarias y socioeconómicas distintas: 1) Pueblo Viejo, en la parte fundacional; 2) Pueblo Nuevo, entre las vías del FCA y las vías del BAP, fue el barrio donde se instalaban los habitantes ingleses que tenían a su cargo la organización y administración de los trenes; y 3) Tierra del Fuego -actual barrio Villa Belgrano- un barrio conformado por empleados no jerárquicos y operarios de los talleres, entre el corredor del BAP y el ejido de Junín (Pompei, 2011). El predio de los talleres ocupa aproximadamente 30 hectáreas, con una superficie construida de unos $100.000 \mathrm{~m}^{2}$ (Dillon, 2010). Cuenta con edificios varios, playa de maniobras y galpones (Figura 3). Constructivamente se compone de una serie de naves industriales de ladrillo a la vista, que albergan cooperativas y emprendimientos varios.

\section{- Edificio de oficinas administrativas}

Situado en las calles Newbery y Sarmiento. En la planta baja funcionaban contaduría, atención al público y la casa del mayordomo. Bajo la escalera se ubicaba una cabina de teléfono para llamadas de corta distancia. En la planta alta estaba la oficina técnica, la oficina del Jefe de Tracción, el cuarto de teléfono para llamadas de larga distancia y la sección de copias heliográficas.

Las fachadas de este edificio se caracterizan por un estilo clasicista y un carácter austero. Presenta muros con un buñado apenas jerarquizado en las esquinas de la planta baja; una sucesión de ventanas - coronadas por guardapolvos rectos - y cornisas sin ornamentos.

\section{- Club Social}

Se ubica en la intersección de las calles Roque Sáenz Peña y Jorge Newbery, frente a la plaza. Inicialmente contaba con habitaciones, baños, un bar, sala para caballeros y sala para damas, un salón, vestíbulos, sala de billares, biblioteca e incluso un teatro. Este club, también conocido como club BAP o Club Inglés, fue fundado a partir de la llegada de los ingleses a Junín. Reunía funcionarios de la compañía ferroviaria y otras personalidades locales. En 1925 se realizó allí un homenaje a los ingleses caídos en la Primera Guerra Mundial, con motivo de la visita del Príncipe de Gales, quien en su viaje a Chile, hizo escala en Junín ${ }^{5}$. 
Figura 3. Edificios ferroviarios patrimoniales (Junín): esquema de localización.

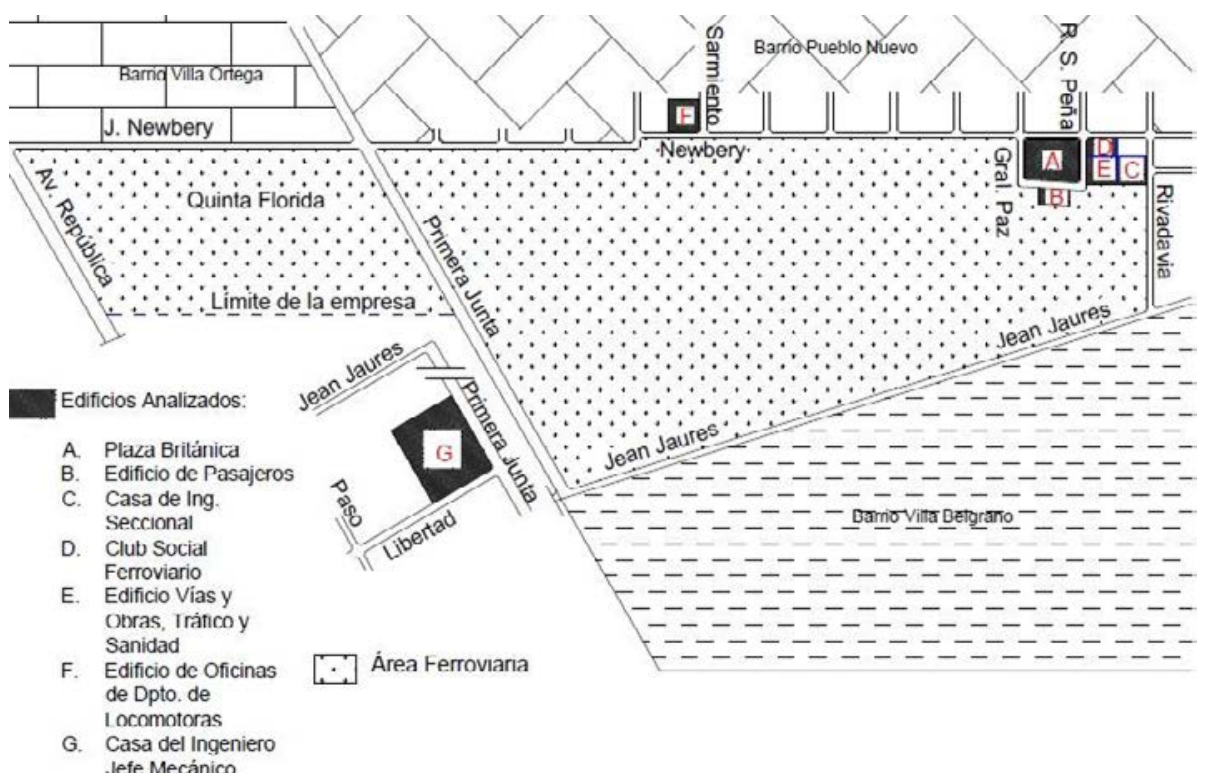

Fuente: Elaboración de los Autores.

\section{- Chalet York y casa del Jefe de Estación}

Se estima que estas viviendas fueron construidas entre 1907 y 1910 para ser ocupadas por el Jefe del Departamento de Mecánica -ingeniero Francis Collin York ${ }^{6}$ y el Jefe de Estación de Junín, respectivamente. Los edificios heredaron el estilo pintoresquista de la tradición británica, con la vivienda exenta en medio del terreno, es decir, sin apoyo de medianeras y con techos inclinados, por lo cual se las conoce con el nombre popular de "chalet". Como otros estilos arquitectónicos europeos de fines del siglo del siglo XIX que fueron replicados en la Argentina, su aplicación sufrió adaptaciones formales y materiales. Sus fachadas estucadas de cemento simulan los elementos decorativos de madera de la arquitectura de tradición pintoresquista; además presentan los desagües pluviales de fundición a la vista (Figura 4).

En las habitaciones principales del Chalet York se destacan hogares a leña, cada uno con una terminación diferente que combinaba hierro, cerámicas y madera; los cielorrasos eran de placas de zinc estampadas y las paredes incorporaban detalles de boiserie. El jardín fue diseñado por la dirección de Parques y Jardines de Junín y contaba con canchas de tenis, cancha de bochas, un pequeño vivero semicubierto y un galpón de materiales (Petraglia, 2012:78)7.

Figura 4. Chalet York y Talleres Ferroviarios (Junín).
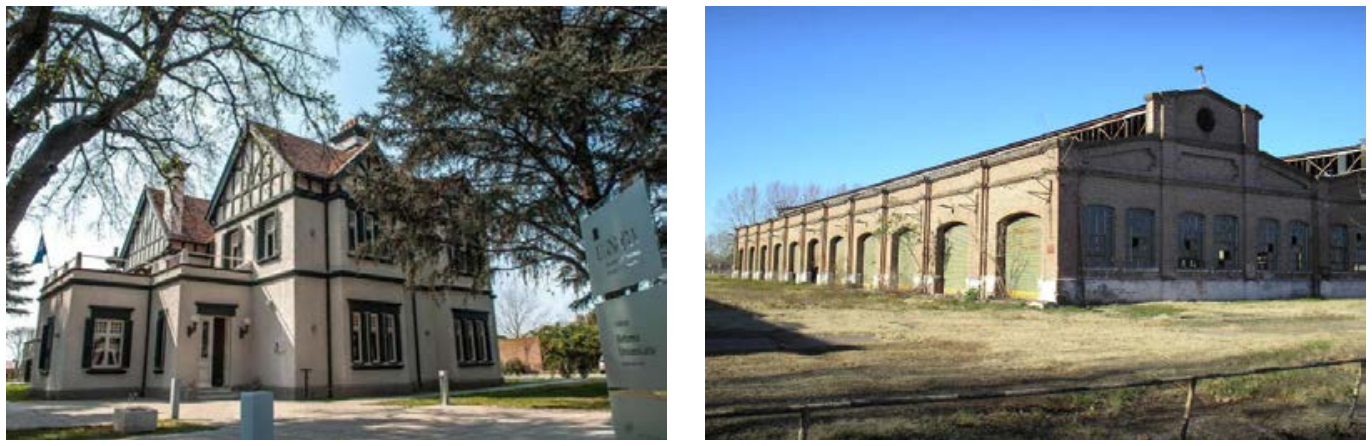

Fuente: Autores. 


\section{- Plaza}

Se ubica entre las calles Newbery, General Paz, Roque Sáenz Peña y el Pasaje La Porteña, sobre los que se extiende la estación. Constituye un espacio organizador de las actividades ferroviarias, ya que en torno a ella se ubican los edificios de la estación, la Casa del Ingeniero Seccional, el Club Social Ferroviario y el Edificio Vías y Obras, Tráfico, y Sanidad. A partir de la instauración del Club Social, se ubicó sobre la plaza una de las canchas de tenis que eran utilizadas por los empleados ferroviarios, aunque en la década de 1930 fue retirada. Esta plaza se mantuvo sin cambios significativos por varias décadas, hasta que para el primer centenario de la llegada del ferrocarril a Junín (1984), se reformó y rebautizó "Plaza Ferrocarriles Argentinos". En 2011 fue remodelada y se colocó la escultura "El origen”, obra de los arquitectos locales Salvador Roselli y Julio Lazcano, realizada con materiales ferroviarios.

\section{- Edificio del ex -Hospital Ferroviario}

El edificio se ubica en Sarmiento y Libertad, en el sector de la ciudad que creció con la actividad y el asiento del personal ferroviario. Tuvo funciones sanitarias para los empleados del ferrocarril y tras la privatización, funcionó allí la Comunidad Terapéutica Ferromed. En 2012 el edificio fue adquirido y rehabilitado por la UNNOBA para actividades académicas.

b) en Mechita:

- Playón Ferroviario:

El predio está compuesto por diversas construcciones (Figura 5). Se ingresa desde Av. Quintana, por el sector administrativo, que consta de tres edificios en buen estado. En el playón ubicado en el lado sur del trazado ferroviario, se ubican los talleres para la reparación de los vehículos. El taller ferroviario es un gran edificio de arquitectura industrial, con ladrillos a la vista y techo con estructura metálica tipo shed. Los vehículos ingresan por un ramal desviado de la red principal. En el interior, las instalaciones son adecuadas para la reparación de vagones y locomotoras, contando con fosas, grúas, herrería y carpintería. La iluminación proviene de ventanas corridas, con carpintería de hierro y vidrios repartidos.

Hacia el Oeste, aproximadamente a 2kilómetros de los talleres, se encuentra el edificio que perteneció al Control de Tráfico Mecha. Allí se distribuía el movimiento de los trenes hacia diferentes ramales. Este inmueble consta de dos pisos, con planta en forma de L. La planta alta se encuentra en estado de abandono y la planta baja está habitada desde hace once años por una familia autorizada por el municipio. Mantiene el estilo con ladrillos a la vista y techos de chapa a dos aguas. En el extremo este del predio está la Estación de Carga y pasajeros, de tamaño reducido, en correspondencia con el diseño de todas las edificaciones de la línea (Catálogo de Bienes Patrimoniales, 2012).

\section{Figura 5. Edificios y sitios patrimoniales (Mechita): esquema de localización.}

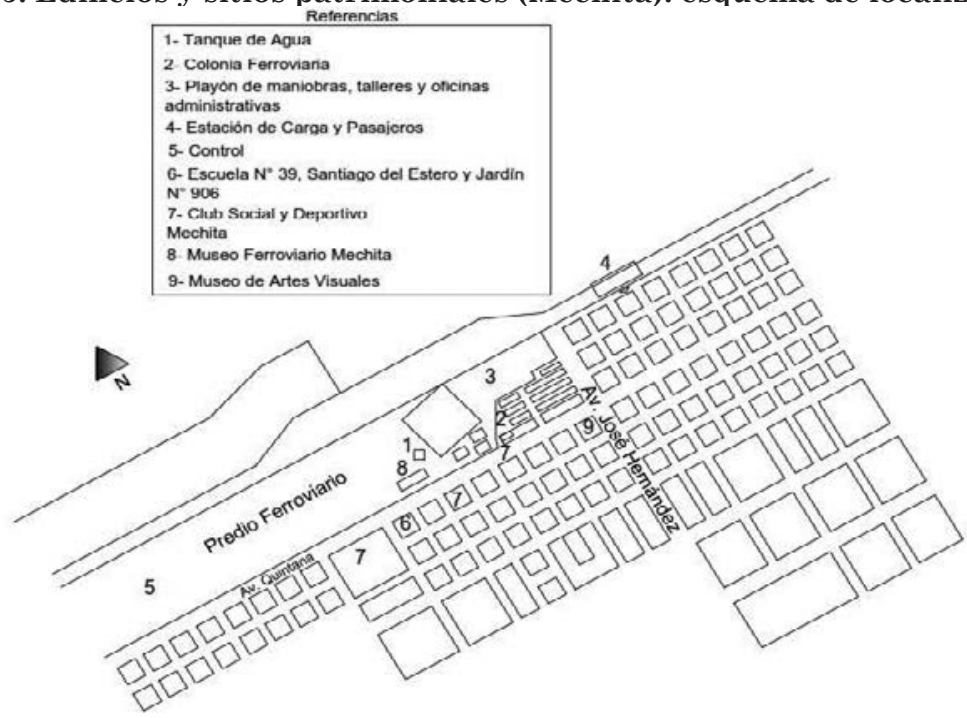

Fuente: Elaboración de los Autores. 


\section{- Colonia:}

La empresa ferroviaria Buenos Aires Western Railway Limited construyó para sus empleados 118 viviendas distribuidas en un barrio de nueve sectores, divididos por estrechos pasajes. Las viviendas destinadas al personal jerárquico se ubicaron sobre la calle principal; presentan elevados techos a dos aguas y gran calidad constructiva. Contaban con dos, tres y cuatro habitaciones, jardín al frente y patio trasero, compartiendo la medianera lateral formando una simetría rebatida. Por su parte, las casas de los operarios - ubicadas hacia las vías - eran bajas, sencillas y pequeñas, compartían la medianera trasera, contando solamente con un patio delantero. Todas presentan una tipología y un estilo general de impronta británica (Figura 6).

En esta colonia se distinguen características comunes a las viviendas ferroviarias: la utilización combinada de tipologías de vivienda jerárquica y en bloques; el crecimiento longitudinal de la estructura urbana del pueblo; la dotación de servicios básicos provistos por la compañía ferroviaria, así como el mantenimiento de las viviendas; y la incorporación de equipamiento deportivo, social y cultural para uso exclusivo de los empleados (Morello y Aguilar, 2006).

Figura 6. Colonia ferroviaria: Viviendas de personal jerárquico y viviendas de operarios.
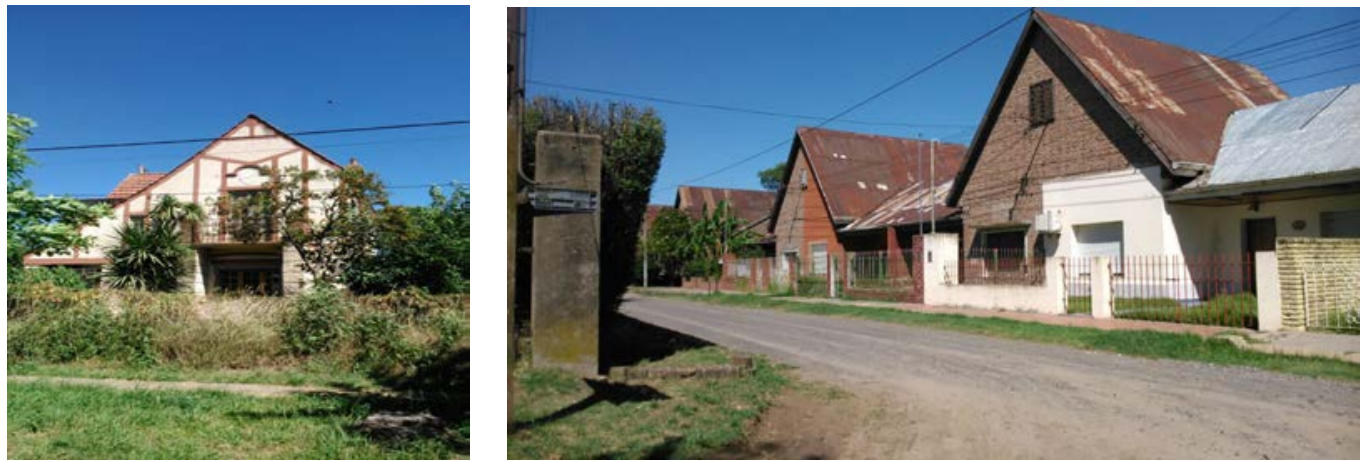

Fuente: Autores.

\section{- Club Social y Deportivo:}

Este edificio se ubica entre la colonia y el predio de los talleres. Presenta dos cuerpos de grandes dimensiones, construido con estructura de hierro, carpintería de madera y cerramientos de chapas. Constituía parte del equipamiento comunitario que la empresa construyó originalmente para la colonia ferroviaria. En 2016 el edificio sufrió un incendio que destruyó gran parte de sus instalaciones. La comunidad se ha agrupado para acelerar la reconstrucción y devolverle su función original.

\section{- Material ferroviario móvil:}

En el sector de los talleres y depósito se encuentran locomotoras y vagones de diferentes épocas ferroviarias. Entre ellos, la más antigua es la locomotora a Vapor Clase 15B 1567, perteneciente a Ferro Club Chivilcoy; una locomotora General Electric U18C 8131, que sirvió en la década de 1970 en la línea Belgrano Sur; el coche a motor Materfer 001, denominado CM001; y vagones Buriasco, que fueron productos nacionales (Figura 7).

\section{- Torre de hierro:}

Para cubrir la demanda de agua en los talleres y la colonia ferroviaria, se instaló una torre de estructura metálica remachada, de 18 metros de altura, con un tanque con capacidad para un millón de litros, rodeado en su base por una plataforma que funcionaba como balcón; y un tanque más pequeño de reserva. Cuando se realizó el tendido de la red pública de agua en la localidad, el tanque quedó en desuso y fue deteriorándose con el tiempo. Por razones de seguridad se desmontó el tanque y solo quedó la torre. Por su relevancia constructiva y visual, ésta fue protegida por ordenanza municipal en $2004^{8}$. 
Figura 7. Predio de los Talleres Ferroviarios Mechita.
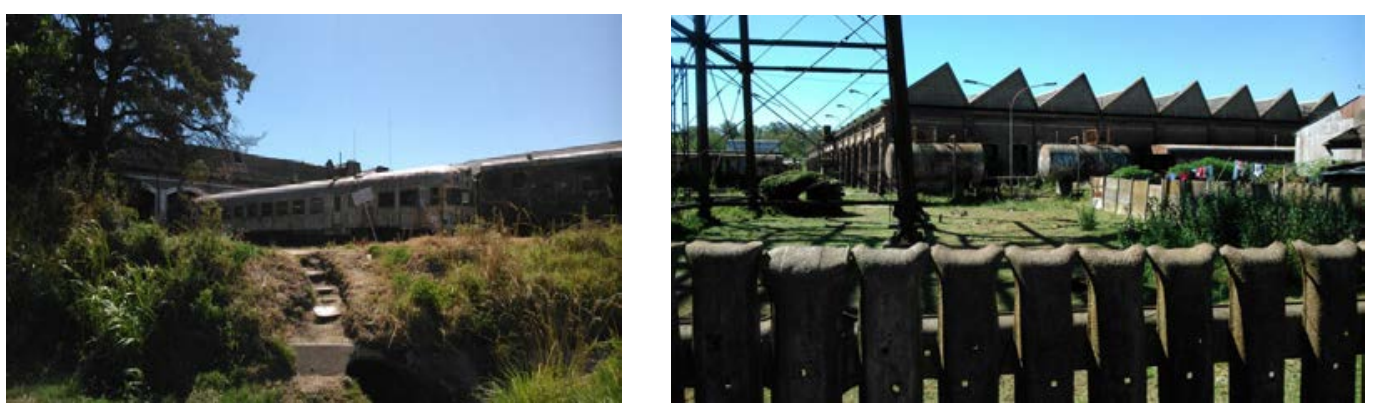

Fuente: Autores.

\section{Alternativas de re-funcionalización}

Los edificios que componen los complejos ferroviarios fueron rehabilitados para desempeñar nuevas funciones. Mientras en otras partes del mundo estos predios se lotean o se destinan a inversiones inmobiliarias y comerciales, en los casos analizados se impuso la actividad cultural, universitaria y turística.

En Junín, el complejo ferroviario fue sectorizado en 1968 en tres talleres, cesando poco después la actividad de reparación de vagones. Esta desafectación dejó como saldo, además de 30 hectáreas casi sin uso, en pleno centro de la ciudad, alrededor de $100.000 \mathrm{~m} 2$ de construcciones de distinto porte, con un valor intrínseco, ya que son ejemplos constructivos de la tradición industrial inglesa (Figura 8). Además existe un amplio patrimonio de arqueología industrial con herramientas, enseres, maquinarias y otros objetos que muestran el desarrollo tecnológico de los talleres (Dillon et al, 1999). Pero fue a partir de principios de la década de 1990, con la privatización de los ferrocarriles, cuando tiene lugar la re-funcionalización de los edificios.

La Universidad y la Municipalidad se constituyeron en dos actores principales del proceso de re-funcionalización de los edificios subsidiarios (oficinas, club, dependencias administrativas, edificio sanitario, etc.), pertenecientes a la compañía ferroviaria original. Los talleres fueron asiento de cooperativas y emprendimientos varios:

- Cooperativa de Trabajo Talleres de Junín (CoTTaJ): creada por un grupo de empleados de los Talleres ante la posibilidad de perder sus fuentes de trabajo. Realiza actividades de reacondicionamiento y mantenimiento de material ferroviario, que incluye vagones, coches motores, vías, terrenos, edificios, galpones, etc.

- Instituto Nacional de Asociativismo y Economía Social (INAES), encargado de legislar, controlar y promover la economía social con respecto a cooperativas y mutuales. En una parte acotada del predio, restaura edificios donde realiza actividades sociales, culturales y deportivas. También sirven como depósitos de maquinarias y para restaurar vagones, que transforman para otros usos.

- Bomberos y policía: poseen un taller en donde se realizan tareas de mantenimiento de camiones y depósito de otros vehículos. También utilizan gran parte de la antigua sección Tornería como depósito Judicial de vehículos secuestrados.

- Estación de Servicio Junín (EDS), que trabaja conjuntamente con la empresa "Trenes Argentinos. Cargas y Logística” de la línea San Martín. Se encarga de realizar el mantenimiento de locomotoras.

A pesar de la presencia de múltiples actores, la mayor parte del predio y galpones de los talleres se encuentran en estado de deterioro edilicio. Por su parte, los edificios subsidiarios del complejo ferroviario tuvieron un destino de rehabilitación y re-funcionalización que ha dinamizado la vida de las zonas urbanas o barrios en los cuales tienen asiento:

- Edificio del Club Social: fue refaccionado en la década de 1980 y a partir de 1990 funcionó como sede del Centro Universitario Regional Junín. En 2003 la UNNOBA instaló allí el Rectorado, hasta su traslado al Chalet York, en 2014. En la actualidad funcionan aulas y actividades administrativas de la Universidad. 
- Edificio de oficinas administrativas: está ocupado por la Escuela de Tecnología de la UNNOBA y por el Museo de Arte Contemporáneo Argentino MACA.

- Hospital Ferroviario: fue rehabilitado como sede académico-administrativa de UNNOBA, a la cual se anexaron nuevos pabellones, laboratorios y auditorio.

- Viviendas del personal jerárquico: pasaron a manos del Estado luego de la estatización de los ferrocarriles en 1947. El chalet York fue donado a la Universidad por el municipio de Junín y funciona como sede del Rectorado de UNNOBA. La casa del jefe de Estación pertenece a la municipalidad y allí funciona el Archivo Histórico y Museo local.

Figura 8. Cooperativas de trabajo en edificios de los ex -talleres ferroviarios (Junín).
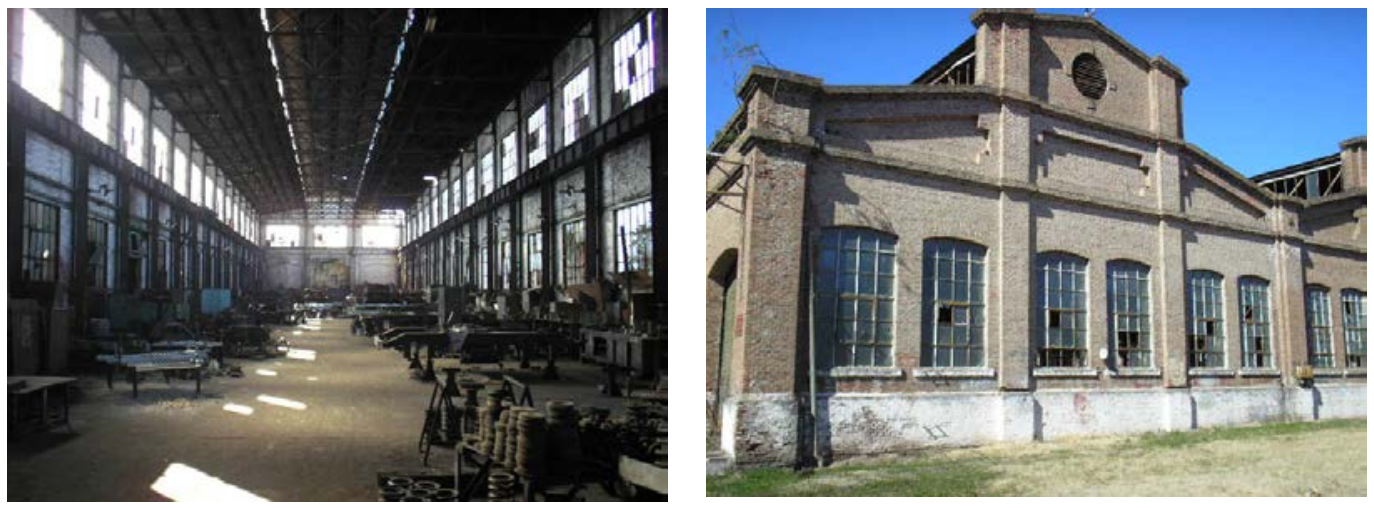

Fuente: Autores.

En Mechita, la re-funcionalización de los talleres se vincula a una experiencia que llevó a cabo el municipio de Bragado entre 2001-2003 ${ }^{9}$ para la puesta en valor del patrimonio cultural local. Luego se firmaron convenios entre el municipio y la Organización No Gubernamental de Promoción y Desarrollo RESPONDE (Recuperación Social de Poblados Nacionales que desaparecen), cuyo objetivo es la recuperación de pueblos en riesgo de desaparición. Posteriormente, se inició el proyecto Potenciar Comunidades, diseñado y gestionado por la Fundación Los Grobo, con la participación de otras empresas y organizaciones ${ }^{10}$, que busca fortalecer la identidad local y promover el desarrollo rural (Presta et al, 2009). Para eso se amplió la estructura edilicia del Museo de Artes Visuales y se promovió la creación de una oficina de turismo, a la vez que se conformó una red de actores locales, encargada de brindar capacitaciones en turismo, diseño y arte a jóvenes de la localidad, con la finalidad de posicionar al pueblo como destino turístico y generar posibilidades de inserción laboral local ${ }^{11}$.

En 2010 en Mechita vivían unos 1.800 habitantes (INDEC 2010) ${ }^{12}$. Si bien los talleres aún trabajan, sólo unas 40 personas están empleadas allí. Por la estación pasan dos trenes diarios de pasajeros, uno en cada sentido del recorrido. La comunidad procuró poner en valor el patrimonio ferroviario y posicionar a Mechita como un referente turístico cultural en relación con la idea de "pueblo ferroviario". Se impulsaron proyectos turístico-culturales rehabilitando los talleres para las siguientes funciones:

- Museo Ferroviario: Se organizó con motivo del aniversario de los 100 años de Mechita, con el objetivo de revalorizar y rescatar el patrimonio histórico ferroviario que dio origen al pueblo. El Gobierno Municipal logró que la empresa concesionaria cediera en comodato las dependencias ferroviarias pertenecientes a la Sub-Usina, que contienen tres edificios instalados en un mismo sector del predio del playón (Figura 9). Los objetos que lo constituyen fueron recuperados del lugar o donados por los vecinos del pueblo. Consta de cuatro salas temáticas, ubicadas en cada inmueble ${ }^{13}$.

- Museo de Artes Visuales: En 2006, con motivo del centenario de la localidad, el escultor Hernán Dompe aceptó realizar una obra totémica con piezas ferroviarias, elegidas por el propio artista, dentro del contexto del enclave ferroviario ligado a la historia del pueblo. Otros artistas visuales también realizaron trabajos in situ, inspirados en distintos aspectos del pueblo, y de esta manera fue surgiendo una colección de obras de arte. Con el apoyo del municipio de Bragado se construyó un espacio inicial para albergar y exhibir las primeras obras plásticas. Más tarde, a partir 
del proyecto de Potenciar Comunidades, la Fundación Los Grobo, la empresa Knauf y Peisa, la Cooperativa de Teléfonos y el Instituto Secundario Mechita, se unieron al proyecto logrando la ampliación del espacio que finalmente sería el Museo de Artes Visuales (MAV). El mentor de este proyecto es el artista plástico mechitense Juan Doffo ${ }^{14}$, quien posee un fuerte vínculo con su lugar de procedencia, expresado a través de su obra. En 2010 se inauguró el MAV, proyecto que permite mostrar en el ámbito cultural bonaerense la obra de consagrados artistas de las artes visuales nacionales.

- Museo privado Recreo Don José: En el año 2005, cuando se inició el proyecto de dar al pueblo una impronta turística, César Giommi armó el Recreo Don José, en homenaje a su padre, donde se exhiben piezas ferroviarias y obras escultóricas de su autoría. Este artista, oriundo de Mechita, fue empleado ferroviario hasta la década de 1990 y heredó el oficio de herrero de su tatarabuelo. Estudió dibujo y pintura, y trasladó ese aprendizaje al diseño de sus esculturas, que construye de forma autodidacta. El museo fue declarado de interés cultural-patrimonial y turístico del partido de Bragado. En 2015 Giommi realizó una reconstrucción del frente de una locomotora, que fue emplazada en el acceso a Mechita, sobre la ruta nacional $\mathrm{N}^{\circ} 5$.

Mechita ofrece variadas atracciones turísticas, culturales y sociales que rescatan su historia y su patrimonio cultural y arquitectónico. El complejo ferroviario está protegido legalmente, por declaratorias municipales y provinciales, para su conservación y puesta en valor ${ }^{15}$.

\section{Figura 9. Museo ferroviario y Club Social (Mechita).}
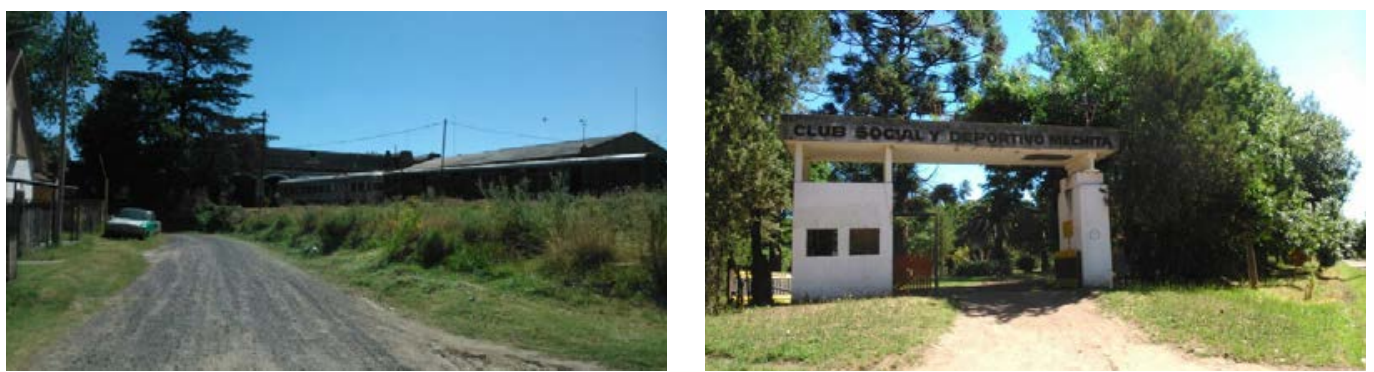

Fuente: Autores.

\section{Consideraciones finales}

En el entresiglos XIX-XX, durante el proceso de territorialización del Noroeste bonaerense, la región consolidó un perfil agropecuario, con el que se ha identificado a lo largo del tiempo. Las actividades ganaderas - primero- y agrícolas -después- se expandieron concomitantemente al tendido de infraestructura.

La extensión de la red ferroviaria hacia el interior de la provincia fue uno de los instrumentos para el fomento a la producción, generando y vinculando circuitos comerciales. Los ramales de las distintas compañías ubicaron sus estaciones en los pueblos y en las estancias, y originaron nuevos puntos de asentamiento a lo largo de su recorrido. La instalación de talleres ferroviarios fortaleció también la actividad económica de las localidades involucradas, otorgándoles, por su envergadura y significado, identidad ferroviaria. De este modo, las redes de transporte para la producción agropecuaria se convirtieron en vehículos de flujos comerciales y económicos hacia los puertos del litoral, pero también generaron conexiones intra-regionales que reforzaron los vínculos internos, ayudando a definir al NOBA como región.

Hacia el último tercio del siglo XX, el desmantelamiento del ferrocarril, la disminución de flujos comerciales y de personas, degradación de la infraestructura y la pérdida de población, conllevaron procesos de des-territorialización con debilitamiento de la identidad social.

La rehabilitación y re-funcionalización del patrimonio favoreció una readaptación social y económica. En Junín, la puesta en valor el patrimonio ferroviario fue realizada por la Universidad, y en menor medida por la municipalidad, con la recuperación de edificios y sitios ferroviarios emblemáticos. En Mechita, programas municipales, la Fundación RESPONDE y el programa Potenciar Comunidades, 
inició un proyecto para el armado de un museo ferroviario y un circuito histórico que busca revitalizar y fortalecer la identidad ferroviaria del pueblo, a través del desarrollo del turismo, el arte y la cultura.

En estos procesos de re-territorialización, las comunidades de perfil agropecuario incorporaron actividades turísticas, universitarias y culturales, enriqueciendo su identidad original. En la región NOBA, un nuevo escenario aparece dinamizado por la rehabilitación y re-funcionalización de espacios y construcciones pertenecientes al ferrocarril. La comunidad se ha adaptado a estos cambios que ella genera, apostando a la valorización del patrimonio ferroviario.

\section{Bibliografía}

Carrizo, Silvina y Yuln, Melina

2014 "Patrimonio Arquitectónico del Noroeste de la Provincia de Buenos Aires. Iniciativas para su valorización”. En XV Encuentro de Economías Regionales del Plan Fénix. Congreso Nacional de

Economía. Buenos Aires: Facultad de Ciencias Económicas, Universidad de Buenos Aires. Dillon, Pedro

2010 Programa Ferrourbano Junín. Web: http://programa-ferrourbano-junin.blogspot.com.ar/ Feria Toribio, J. M.

2010 "Patrimonio territorial y desarrollo sostenible: un estudio comparativo en Iberoamérica y España".

Estudios Geográficos, 71(268), 129-159. Consultado el 20 de marzo de 2015. doi:10.3989/estgeogr.0472

Franco, Laura y Dillon, Pedro

1998Proyecto Ferro-Urbanístico. Junín: Municipalidad de Junín.

Galindo González, J., Y Sabaté Bel, J.

2009 "El valor estructurante del patrimonio en la transformación del territorio". Apuntes, 22(1), 20-33.

Consultado en: http://dialnet.unirioja.es/servlet/articulo?codigo $=3150331$

Haesbaert, Rogerio

2005 "Da desterritorialização a multiterritorialidade" Anais Do X Encontro de Geógrafos da América

Latina. Universidade de São Paulo

Iácullo, A. Y Tarullo, R.

2013 Ferroviarios. Nuestra historia. Junín: CEDI UNNOBA.

Montecelli Federico; Carrizo, Silvina y Yuln, Melina

2015 "El ferrocarril en Junín (provincia de Buenos Aires). Una lectura histórica a partir de planos arquitectónicos e ingenieriles". En $4^{\circ}$ Congreso Iberoamericano y XI Jornada Técnicas de Restauración y Conservación del Patrimonio. La Plata: CIC-LEMIT

Morello, A. Y Aguilar, G.

2006 "La lógica del sistema ferroviario como germen para concebir un proyecto de rehabilitación integral del patrimonio. Los casos de mechita y colonia sola, dos experiencias para la recuperación de viviendas ferroviarias". Congreso Internacional Patrimonio Industrial, Puesta en valor del Patrimonio

Industrial. Sitios, Museos y casos. Chile. http://www.morelloyaguilar.com/espanol/documentos/La\%20 logica\%20del\%20sistema\%20ferroviario.pdf

Petraglia, Pablo G.

2012 El chalet de Mr. York. Junín: Universidad Nacional del Noroeste de la Provincia de Buenos Aires. Pompei, Ana Laura

2001 Historia de los talleres Ferroviarios de Junín. Desde su creación a la conformación de la Cooperativa de Trabajo Talleres Junín (1886-1994). Tesis de Licenciatura en Historia, UNNOBA (inédita).

Presta, Susana; Landaburu, Liliana Y Figueira, Patricia

2009 "Desenvolvimento do turismo cultural e as políticas de desenvolvimento local. Análise de Caso". Revista Aval Año 2 (3) n 3-4 "JAN-DEZ" http://www.mapp.ufc.br/images/revista_aval/edi\%C3\%A7\%C3\%B5es/7d/ PG_37-46_ARTIGO_4.pdf

Sabaté Bel, J.

2005 "De la preservación del patrimonio a la ordenación del paisaje". En Identidades: Territorio, Cultura, Patrimonio, 1(Ensayos), 15-33. Consultado en: http://hdl.handle.net/2099/1885

- 2014 "Turismo, paisaje y urbanismo: un diálogo necesario". ACE Architecture, City and Environment, 9(25), 279-302. Consultado en: doi:10.5821/ace.9.25.3627. 
Sabaté Bel, J., Y Benito Del Pozo, P.

2010 "Paisajes culturales y proyecto territorial: un balance de treinta años de experiencia". Identidades:

Territorio, Cultura, Patrimonio, 2 (Ensayos), 2-21. Consultado el 20 de marzo de 2015 http://hdl. handle.net/2099/10716

Sagastume, Ana L.

2013Imaginarios y memorias de ex-ferroviarios que trabajaron en Ferrocarriles Argentinos. Junin: 1948-1993, Tesis de maestría de la Universidad CAECE (inédita).

Taghón A.; Manzione G.; Yuln, M.; Carrizo, S.; Lima, L.; Montecelli, F.

2015 "Rescate de bienes patrimoniales relacionados con el ferrocarril en la provincia de Buenos Aires: Junín, talleres de la compañía BAP. Mechita, pueblo ferroviario, Ferrocarril Oeste". En Jornadas de Patrimonio Industrial. San Juan: Facultad de Arquitectura, Urbanismo y Diseño. Universidad Nacional de San Juan.

Troitiño, M. A.

1998 "Patrimonio Arquitectónico, Cultura y Territorio", en Ciudades: Revista Del Instituto Universitario de Urbanística de La Universidad de Valladolid, 4, 95"104. Consulado en: http://dialnet.unirioja.es/ servlet/articulo? codigo $=2241051$

Yuln, Melina; Carrizo, Silvina; Lima, Luis; Taghón, Alejandra; Manzione, Graciela

2013 "Estudios y Gestiones sobre Patrimonio arquitectónico en el Noroeste de la Provincia de Buenos Aires". En $3^{\circ}$ Congreso Iberoamericano y XI Jornada Técnicas de Restauración y Conservación del Patrimonio. La Plata: CIC-LEMIT

- 2014 "Patrimonio arquitectónico en la región del noroeste de la provincia de Buenos Aires. Su conformación a través de la expansión ferroviaria y la electrificación”. En IV Seminario Internacional de Patrimonio Agroindustrial. Recursos para el desarrollo. San Miguel de Tucumán: Instituto de Historia y Patrimonio. Facultad de Arquitectura. Universidad Nacional de Tucumán.

\section{Notas}

1 Censo Nacional de 1895

2 La locación surgió por una discrepancia entre la empresa Buenos Aires Western RailwayLimited, de capitales ingleses, y los dueños de los terrenos donde se ubicarían los talleres en la ciudad de Bragado. Al no llegar a un acuerdo comercial entre ambos, el Presidente Manuel Quintana ofreció donar terrenos de su propiedad, pertenecientes a la Estancia Los Manantiales, para destinarlos a tal fin.

3 Nombre que recibe en agradecimiento al Dr. Manuel Quintana y que correspondiera a su nieta. Por su parte, la zona en donde se ubicó la oficina de Control de Tráfico, recibió el nombre de Mecha por la esposa de Quintana.

4 El ex -ferrocarril BAP contaba en 1970 con tres talleres de trocha ancha: Alianza (Santos Lugares, Provincia de Buenos Aires), Junín y Mendoza capital. El ex-ferrocarril del Oeste, contaba con los talleres de Liniers y Villa Luro, Provincia de Buenos Aires.

5 Revista Caras y Caretas, 1925.

6 Conocido como Mr. York, habría llegado a Argentina en 1885 y regresado a Inglaterra en 1916. Trabajaba en el edificio del Departamento de Locomotoras. Participó en la construcción de obras civiles juninenses, como la construcción del Palacio Municipal o el puente de calle Lartigau (Petraglia, 2012).

$7 \quad$ Un dato que destaca Petraglia (2012) es la orientación de los locales del Chalet York respecto del asoleamiento, ya que responden a una implantación propia del hemisferio norte, con lo cual se estima que los planos fueron proyectados en el Reino Unido.

8 Decreto 1415, Ordenanza $\mathrm{N}^{\circ}$ 3196/04. Declara de interés cultural la Torre de hierro donde se asentó el primer tanque de agua de la localidad de Mechita, ubicada en el predio del actual depósito de locomotoras.

9 Para ello se diseñó el Programa Municipal Identidad y Patrimonio (PROMIP) con intervenciones en las localidades rurales (O’Brien, Mechita, Comodoro Py y Warnes) y en la ciudad cabecera del partido. Las acciones de este Programa se articulaban desde el Archivo Histórico Municipal y se clasificaban en 1) acciones de promoción; mediante jornadas y seminarios en las distintas localidades; 2) acciones de relevamiento, a través de encuestas y entrevistas con actores locales y 3) acciones de Información, con la construcción de un Sistema de Información del Patrimonio Cultural (SIPAC) (Abarzúa, 2004).

10 Fundación Acindar, Fundación Navarro Viola, Fundación Diario La Nación, Grupo Clarín, BASF Argentina, El Tejar, Banco Galicia, Editorial Troquel, Monsanto, Universidad Católica Argentina y Universidad de San Andrés.

11 http://www.potenciar.org.ar/

12 Datos del Censo 2010.

13 Sala A: "La historia del Ferrocarril del Oeste", "Mechita: origen y progreso", "Del vapor al diesel". Sala B: "Comunidad mechitense y trabajo ferroviario". Sala C: Material de uso ferroviario. Sala D: Caldera Zerolit.

14 Juan Doffo, 25/07/1948. Su currículo incluye múltiples exposiciones colectivas e individuales, en el país y en el exterior. Obras de su autoría se encuentran en varios museos de mundo.

15 Decreto 1415, Ordenanza $\mathrm{N}^{\circ}$ 3196/04. Declara de interés cultural la Torre de hierro donde se asentó el primer tanque de agua de la localidad de Mechita, ubicada en el predio del actual depósito de locomotoras; Decreto $\mathrm{N}^{\circ} 741$.Ordenanza $\mathrm{N}^{\circ}$ 
3256/05. Declara Sitio Histórico Cultural a la Villa ferroviaria "La Colonia" de la localidad de Mechita, Cuartel III del Partido de Bragado; Ordenanza N 3307 / 05 Declara Pueblo Histórico a la localidad de Mechita Cuartel III, del Partido de Bragado; Ley 13607 (2006). La Legislatura de la Provincia de Buenos Aires declara Sitio de Interés Histórico Territorial, incorporado al Patrimonio Cultural de la Provincia de Buenos Aires, a todo el Conjunto Ferroviario ubicado en el predio del ex Ferrocarril Sarmiento, actual depósito de locomotoras de la localidad de Mechita, que incluye los talleres del ex ferrocarril Sarmiento, el barrio de viviendas, la torre de hierro donde se instaló el primer tanque de agua, edificios y construcciones complementarias anexas al ferrocarril; Ordenanza $N^{\circ} 3419 / 06$. Declara de Interés Cultural-Patrimonial y turístico del Partido de Bragado, al emprendimiento privado "Recreo Don José"; Ley 14320 (2011). La legislatura bonaerense declara de Interés Cultural Provincial al Museo de Artes Visuales. 